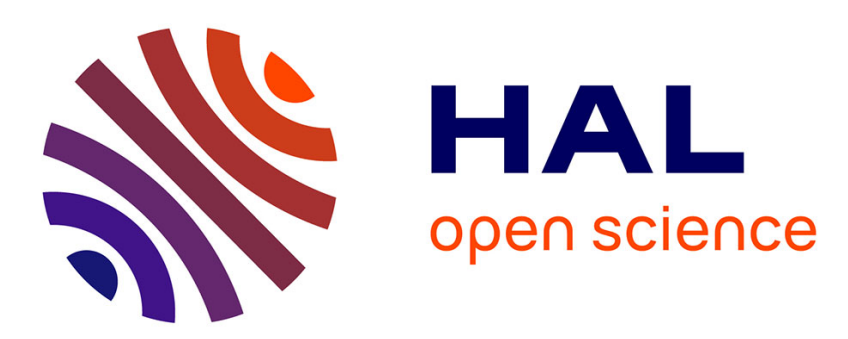

\title{
Une transformation du problème d'élasticité linéaire en vue d'application au problème de l'inclusion et aux fonctions de Green
}

Ahmad Pouya

\section{- To cite this version:}

Ahmad Pouya. Une transformation du problème d'élasticité linéaire en vue d'application au problème de l'inclusion et aux fonctions de Green. C.R. Acad. Sci. Paris, 2000, t 328 (Série II b), pp.437-443. hal-00636031

\section{HAL Id: hal-00636031 \\ https://hal.science/hal-00636031}

Submitted on 26 Oct 2011

HAL is a multi-disciplinary open access archive for the deposit and dissemination of scientific research documents, whether they are published or not. The documents may come from teaching and research institutions in France or abroad, or from public or private research centers.
L'archive ouverte pluridisciplinaire $\mathbf{H A L}$, est destinée au dépôt et à la diffusion de documents scientifiques de niveau recherche, publiés ou non, émanant des établissements d'enseignement et de recherche français ou étrangers, des laboratoires publics ou privés. 
C.R. Acad. Sci. Paris, t. 328, Série II b, p. 437-443, 2000

Mécanique des solides et des structures / Mechanics of solids and structures

\title{
Une transformation du problème d'élasticité linéaire en vue d'application au problème de l'inclusion et aux fonctions de Green
}

\author{
Ahmad POUYA \\ G3S-LMS, Ecole Polytechnique, 91128, Palaiseau Cedex
}

\begin{abstract}
Résumé : Nous présentons une transformation simple du problème de structure élastique linéaire. Le problème transformé se présente sous forme d'un nouveau problème de structure élastique linéaire avec un comportement, une géométrie et des données de forces et de déplacements imposés différents. Le problème transformé peut être plus simple à traiter ou correspondre à des cas de solutions analytiques connues. A l'aide de cette transformation, on peut ramener le problème de l'inclusion ellipsoïdale en un problème d'inclusion sphérique, étendre les résultats connus pour le tenseur d'Eshelby pour les cas de matrice isotrope ou d'isotropie transverse, à des cas plus larges, et enfin, établir l'expression analytique de la fonction de Green du milieu infini pour des cas de comportements orthotropes plus riches que l'isotropie transverse, ainsi que des cas non orthotropes.
\end{abstract}

Elasticité linéaire / inclusion / fonction de Green

\section{A transformation of the problem of linear elastic structure for application to the inclusion problem and to Green functions}

\begin{abstract}
A simple transformation of the problem of the linear elastic structure is presented. The transformed problem corresponds to a new problem of linear elastic structure with different behaviour, geometry and prescribed forces and displacements. The transformed problem can be easier to study, or can correspond to cases with well-known solutions. By means of this transformation, the problem of ellipsoidal inclusion is transformed into a problem of spherical inclusion, the analytical results known for the Eshelby tensor for an isotropic or transversely isotropic matrix are extended to more general cases of matrix behaviour, and finally, close form expressions of the Green function for an infinite medium are derived for some cases of elastic behaviour without transversal isotropy or orthotropy.
\end{abstract}

Linear Elasticity / inclusion / Green's function

Abridged English version : Consider a linear elastic solid occupying a volume $\Omega$ in $\mathrm{R}^{3}$. Let us assume that the boundary $\partial \Omega$ is regular and denote $\underline{\mathrm{n}}(\underline{\mathrm{x}})$ the outward normal to $\partial \Omega$ at the point $\underline{x} \in \partial \Omega$. The elastic stiffness tensor $\underline{\underline{\underline{C}}}$ in Cartesian coordinates exhibits the symmetry properties (1) and is positive-definite. The solid is submitted to body forces $\underline{F}(\underline{x})$, to surface tensions $\underline{T}(\underline{x})$ on a part of $\partial \Omega$ denoted $\partial_{\mathrm{T}} \Omega$, and to given displacements $\underline{u}^{\mathrm{d}}(\underline{\mathrm{x}})$ on the complementary part of $\partial \Omega$, denoted $\partial_{\mathrm{u}} \Omega$. The problem consists in finding 
the displacement field $\underline{\mathrm{u}}(\underline{\mathrm{x}})$ verifying equations (3) to (5). The strain and stress fields for this problem are denoted respectively $\underline{\underline{\varepsilon}}(\underline{\mathrm{x}})$ and $\underline{\underline{\sigma}}(\underline{\mathrm{x}})$.

Now, let $\underline{\underline{P}}$ be a given symmetric and positive-definite tensor; we make the changes of variables (6) and (7). The new variable $\underline{\tilde{x}}$ defines a region $\tilde{\Omega}$ in $R^{3}$. The unit outward normal to the boundary $\tilde{\partial} \tilde{\Omega}$ is given by $\underline{\tilde{n}}(\underline{\tilde{x}})=\kappa(\underline{x}) \underline{\underline{P}} \cdot \underline{n}(\underline{x})$ where $\kappa(\underline{x})=\|\underline{\underline{P}} \cdot \underline{n}(\underline{x})\|^{-1}$, $\|\cdot\|$ representing the Euclidian norm. The equations of the problem in terms of $\underline{\tilde{x}}$ and $\underline{\tilde{u}}$ are

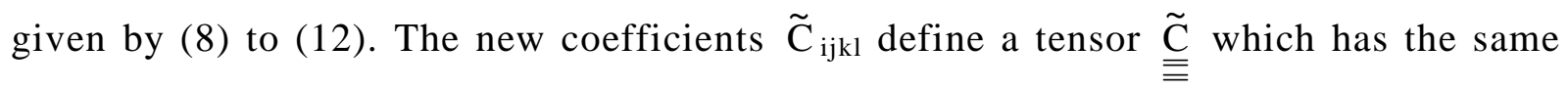
properties of symmetry and positive-definiteness as $\underline{\underline{\underline{\underline{C}}}}$. These equations define a new problem of a linear elastic body having a different geometry and behaviour and submitted to different forces and displacements. The strain and stress fields of this transformed problem are related to those of the initial one by (13).

This transformation can be applied to different problems of elasticity in order to extend the results obtained for some cases of geometry or behaviour to new cases. Examples are given in the following.

$\underline{\text { Elastic Inclusions }}$ - Consider an elastic inclusion $\Omega$ with stiffness tensor $\stackrel{\text { c embedded in }}{\equiv}$ an infinite elastic matrix $\mathrm{M}$ with stiffness tensor $\underline{\underline{\underline{C}}}$. Let us assume that the inclusion has been submitted to a «stress-free» strain $\underline{\underline{\varepsilon}}^{\underline{\underline{\underline{0}}}}$ (Eshelby [1]) deriving from the displacement $\underline{u}^{0}$, and that the matrix undergoes a uniform strain $\underline{E}^{\infty}$ at infinity. The problem consists in finding the displacement fields $\underline{u}^{(1)}$ and $\underline{u}^{(2)}$, defined respectively in $\Omega$ and $\mathrm{M}$, and verifying equations (14) to (18). If $\Omega$ is ellipsoidal, $\underline{\varepsilon}^{0}$ uniform in $\Omega$ and $\underline{\underline{E}}^{\infty}=0$, then the strain in the inclusion is given by (19). Closed-form expressions involving elliptic integrals are known for the components of the "Eshelby tensor" $\underset{\equiv}{\underline{S}}$ for the cases of isotropic or transversely isotropic behaviour of the matrix ([1],[2],[3]). The transformation defined above changes this problem into an equivalent inclusion problem and the relationship (20) is established between the Eshelby tensors of the two problems. This enables one to extend these results known for the Eshelby tensor to new cases of matrix behaviour : those which can be obtained by the transformation (12) from the isotropic or transversely isotropic behaviours.

The transformation of the inclusion problem can also be made in order to change the ellipsoidal form of the inclusion into the spherical form, which can simplify some analytical or numerical calculations.

Transformed cases of the isotropic behaviour - Let us consider a linear isotropic behaviour represented by the tensor $\underset{\underline{\underline{\underline{C}}}}{\underline{\tilde{C}}}$ depending on the Lamé coefficients $\tilde{\lambda}$ and $\tilde{\mu}$. 
This behaviour can be considered as the result of the transformation (12) applied to the elastic behaviour defined by $\mathrm{C}_{\mathrm{ijk}}$ given by (22).

In a system of axes where $\underline{\underline{P}}$ is diagonal with diagonal terms $\mathrm{a}^{\alpha}(\alpha=1,2,3)$, the elastic compliance matrix corresponding to (22) is given by (25) in which the 4 independent parameters $\left(c_{11}, c_{22}, c_{33}, \eta\right)$ are given by (23) and (24). This defines a family of orthotropic materials which have three different moduli in three different directions. Sometimes the models of this family can constitute a good approximation for some anisotropic materials (see table 1). The results known for the Eshelby tensor and for Green functions can be extended to this family.

Green functions - The relationship (28) can be established between the Green functions corresponding to the elastic tensors $\underline{\underline{\underline{\underline{C}}}}$ and $\underline{\underline{\underline{\underline{C}}}}$. This enables one to extend, for instance, the well-known Green function solution for the isotropic behaviour, to the behaviour defined by (25). The solution is given by (31) in which $\rho(\underline{x})=\sqrt{\underline{x} \cdot \underline{\underline{D}} \cdot \underline{x}}, \quad \underline{\mathrm{D}}$ is the diagonal tensor in the reference axes with diagonal terms $\left(a_{\alpha}\right)^{-2}$, and $a_{\alpha}, \tilde{\mu}$ and $\tilde{v}$ are given by (29).

In the same way, the transformation of the Green function solutions ([5],[6],[3]), and also of the displacement potential solutions [7], for the general case of transverse isotropy, leads to solutions for more general cases of orthotropic behaviour, as well as some cases of non orthotropic behaviour.

\section{Problème de départ}

Considérons un corps élastique linéaire occupant un domaine $\Omega$ de $\mathrm{R}^{3}$, de frontière régulière notée $\partial \Omega$. On note $\underline{\mathrm{n}}(\underline{\mathrm{x}})$ la normale unitaire extérieure à $\Omega$ au point $\underline{\mathrm{x}}$. Le

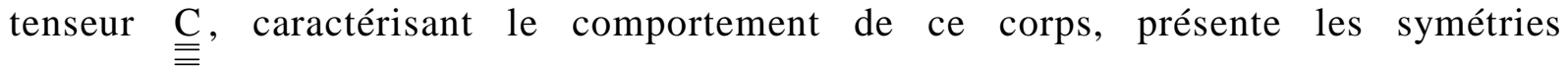
suivantes dans une base orthonormée de $\mathrm{R}^{3}$ :

$$
\mathrm{C}_{\mathrm{ijkl}}=\mathrm{C}_{\mathrm{ijlk}}=\mathrm{C}_{\mathrm{klij}}
$$

Ce tenseur est par ailleurs supposé défini positif :

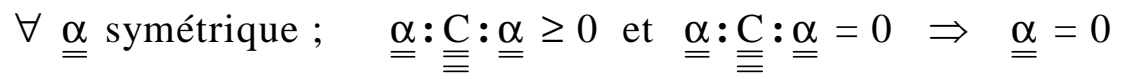

Supposons que ce corps soit soumis à des forces volumiques $\underline{F}(\underline{x})$, à des forces surfaciques $\underline{\mathrm{T}}(\underline{\mathrm{x}})$ sur une partie $\partial_{\mathrm{T}} \Omega$ de $\partial \Omega$ et à des déplacements imposés $\underline{u}^{\mathrm{d}}(\underline{\mathrm{x}})$ sur la partie complémentaire notée $\partial_{\mathrm{u}} \Omega$. Le problème consiste à trouver un champ de déplacement $\underline{\mathrm{u}}(\underline{\mathrm{x}})$ vérifiant les conditions suivantes :

$$
\begin{array}{ll}
\left(\mathbf{E}_{1}\right): & \forall \underline{\mathrm{x}} \in \Omega ; \quad \mathrm{C}_{\mathrm{ijk}} \partial_{\mathrm{jk}} \mathrm{u}_{1}(\underline{\mathrm{x}})+\mathrm{F}_{\mathrm{i}}(\underline{\mathrm{x}})=0 \\
\left(\mathbf{E}_{2}\right): & \forall \underline{\mathrm{x}} \in \partial_{\mathrm{u}} \Omega ; \underline{\mathrm{u}}(\underline{\mathrm{x}})=\underline{\mathrm{u}}^{\mathrm{x}}(\underline{\mathrm{x}}) \\
\left(\mathbf{E}_{3}\right): & \forall \underline{\mathrm{x}} \in \partial_{\mathrm{T}} \Omega ; \mathrm{n}_{\mathrm{j}}(\underline{\mathrm{x}}) \mathrm{C}_{\mathrm{ijk} \mathrm{j}} \partial_{\mathrm{k}} \mathrm{u}_{\mathrm{l}}=\mathrm{T}_{\mathrm{i}}(\underline{\mathrm{x}})
\end{array}
$$


On notera $\underline{\underline{\varepsilon}}$ la déformation dérivant de $\underline{\underline{u}}$ et $\underline{\underline{\sigma}}=\underline{\underline{\underline{C}}}: \underline{\underline{\varepsilon}}$, la contrainte.

\section{Problème transformé}

Considérons un tenseur $\underline{\underline{P}}$ symétrique et à valeurs propres strictement positives, et faisons les changements de variable et de fonction inconnue suivants dans le problème ci-dessus :

$$
\begin{aligned}
& \underline{\mathrm{x}}=\underline{\mathrm{P}} \cdot \underline{\tilde{\mathrm{x}}} \\
& \underline{\tilde{\mathrm{u}}}=\underline{\underline{\mathrm{P}}} \cdot \underline{\mathrm{u}}
\end{aligned}
$$

La variable $\underline{\tilde{x}}$ parcourt un domaine de $\mathrm{R}^{3}$ noté $\tilde{\Omega}$ de frontière $\tilde{\partial} \tilde{\Omega}$. Le vecteur normal unitaire sortant de $\partial \tilde{\Omega}$ est donné par $\underline{\tilde{\tilde{}}}(\underline{\tilde{x}})=\kappa(\underline{\mathrm{x}}) \underline{\underline{\mathrm{P}}} \cdot \underline{\mathrm{n}}(\underline{\mathrm{x}})$ avec $\kappa(\underline{\mathrm{x}})=\|\underline{\underline{\mathrm{P}}} \cdot \underline{\mathrm{n}}(\underline{\mathrm{x}})\|^{-1}, \quad\|\cdot\|$ représentant la norme euclidienne.

Introduisons les nouvelles variable et inconnue $\underline{\tilde{x}}$ et $\underline{\tilde{u}}$ dans les équations $\left(\mathbf{E}_{\mathbf{1}}\right),\left(\mathbf{E}_{\mathbf{2}}\right)$ et $\left(\mathbf{E}_{\mathbf{3}}\right)$ et multiplions les deux membres de $\left(\mathbf{E}_{\mathbf{1}}\right)$ et de $\left(\mathbf{E}_{\mathbf{3}}\right)$ par:

Nous obtenons :

$$
\underline{\underline{Q}}=\underline{\underline{P}}^{-1}
$$

$$
\begin{aligned}
& \left(\tilde{\mathbf{E}}_{1}\right): \forall \underline{\tilde{x}} \in \tilde{\Omega} ; \widetilde{\mathbf{C}}_{\mathrm{ijkl}} \tilde{\partial}_{\mathrm{jk}} \tilde{\mathbf{u}}_{1}(\underline{\tilde{x}})+\widetilde{\mathrm{F}}_{\mathrm{i}}(\underline{\tilde{x}})=0 \\
& \left(\tilde{\mathbf{E}}_{2}\right): \forall \underline{\tilde{x}} \in \tilde{\partial}_{\mathrm{u}} \tilde{\Omega} ; \underline{\tilde{u}}(\underline{\tilde{x}})=\underline{\tilde{u}}^{\mathrm{d}}(\underline{\tilde{x}}) \\
& \left(\tilde{\mathbf{E}}_{3}\right): \forall \underline{\tilde{x}} \in \tilde{\partial}_{\mathrm{T}} \tilde{\Omega} ; \tilde{\mathrm{n}}_{\mathrm{j}}(\underline{\tilde{x}}) \tilde{\mathrm{C}}_{\mathrm{ijk} 1} \tilde{\partial}_{\mathrm{k}} \tilde{\mathrm{u}}_{1}=\widetilde{\mathrm{T}}_{\mathrm{i}}(\underline{\tilde{x}})
\end{aligned}
$$

où on a noté :

$$
\tilde{\partial}_{i}(.)=\frac{\partial}{\partial \tilde{x}_{i}}(.),
$$

$$
\underline{\tilde{F}}(\underline{\tilde{x}})=\underline{\underline{Q}} \cdot \underline{F}(\underline{x}), \quad \underline{\tilde{u}}^{d}(\underline{\tilde{x}})=\underline{P} \cdot \underline{u}^{d}(\underline{x}), \quad \underline{\tilde{T}}(\underline{\tilde{x}})=\kappa(\underline{x}) \underline{\underline{Q}} \cdot \underline{T}(\underline{x})
$$

et où $\underline{\underline{\underline{\underline{C}}}}$ est défini par :

$$
\tilde{\mathrm{C}}_{\mathrm{ijk} \mathrm{l}}=\mathrm{Q}_{\mathrm{im}} \mathrm{Q}_{\mathrm{jn}} \mathrm{C}_{\mathrm{mnpq}} \mathrm{Q}_{\mathrm{pk}} \mathrm{Q}_{\mathrm{q} 1}
$$

On vérifie que $\underset{\underline{\underline{\underline{C}}}}{\widetilde{\widetilde{C}}}$ possède les propriétés de symétrie suivantes :

$$
\tilde{\mathrm{C}}_{\mathrm{ijkl}}=\tilde{\mathrm{C}}_{\mathrm{ijlk}}=\tilde{\mathrm{C}}_{\mathrm{klij}}
$$

et qu'il est défini positif. Le problème transformé se présente donc sous forme d'un nouveau problème de structure élastique linéaire, c'est-à-dire s'exprimant avec exactement les mêmes équations, mais correspondant à des données de géométrie, de comportement et de forces et déplacements imposés différentes. En notant $\underset{\underline{\varepsilon}}{\widetilde{\varepsilon}}$ la déformation et $\underline{\underline{\tilde{\sigma}}}=\underline{\underline{\underline{\underline{C}}}}: \underline{\underline{\tilde{\varepsilon}}}$, la contrainte correspondantes à ce problème, on vérifiera les relations suivantes : 


$$
\underline{\underline{\tilde{\varepsilon}}}(\underline{\tilde{x}})=\underline{\underline{P}} \cdot \underline{\underline{\varepsilon}}(\underline{\mathrm{x}}) \cdot \underline{\underline{\mathrm{P}}}, \quad \underline{\underline{\tilde{\sigma}}}(\underline{\tilde{\tilde{x}}})=\underline{\underline{\mathrm{P}}}^{-1} \cdot \underline{\underline{\sigma}}(\underline{\mathrm{x}}) \cdot \underline{\underline{P}}^{-1}
$$

Le problème transformé peut être plus intéressant à étudier que le problème de départ du fait qu'il peut correspondre à une géométrie ou à un comportement plus simple ou à des cas de solutions analytiques connues. Nous en montrons des exemples dans la suite.

\section{Application au problème de l'inclusion}

Considérons une inclusion élastique de comportement donné par $\underline{\underline{\equiv}}$ dans une matrice élastique homogène et infinie de comportement donné par $\underline{\underline{\underline{\underline{C}}}}$. L'inclusion occupe un sous-domaine borné $\Omega$ de $\mathrm{R}^{3}$ de frontière régulière notée $\partial \Omega$, et la matrice occupe la partie complémentaire, notée $\mathrm{M}$. On suppose que l'inclusion a subi une «déformation libre » au sens d'Eshelby [1] avant d'être insérée dans la matrice. Cette déformation, notée $\underline{\underline{\varepsilon}}^{0}$, peut être non uniforme; on suppose qu'elle dérive d'un déplacement $\underline{u}^{0}$ défini dans $\Omega$. On suppose que la matrice subit à l'infini une déformation uniforme $\underline{E}^{\infty}$. Le problème consiste à trouver les champs de déplacement $\underline{\mathbf{u}}^{(1)}$ dans $\Omega$ et $\underline{\mathbf{u}}^{(2)}$ dans $\mathrm{M}$ vérifiant les conditions suivantes :

$$
\begin{array}{lll}
\text { 1. } & \forall \underline{\mathrm{x}} \in \Omega ; & \mathrm{c}_{\mathrm{ijkl}} \partial_{\mathrm{jk}}\left[\mathrm{u}_{1}^{(1)}(\underline{\mathrm{x}})-\mathrm{u}_{1}^{0}(\underline{\mathrm{x}})\right]=0 \\
\text { 2. } & \forall \underline{\mathrm{x}} \in \mathrm{M} ; & \mathrm{C}_{\mathrm{ijkl}} \partial_{\mathrm{jk}} \mathrm{u}_{1}^{(2)}(\underline{\mathrm{x}})=0 \\
\text { 3. } & \forall \underline{\mathrm{x}} \in \partial \Omega ; & \underline{\mathrm{u}}^{(1)}(\underline{\mathrm{x}})=\underline{\mathrm{u}}^{(2)}(\underline{\mathrm{x}}) \\
\text { 4. } & \forall \underline{\mathrm{x}} \in \partial \Omega ; & \mathrm{n}_{\mathrm{j}}(\underline{\mathrm{x}}) \mathrm{c}_{\mathrm{ijk}} \partial_{\mathrm{k}}\left[\mathrm{u}_{1}^{(1)}(\underline{\mathrm{x}})-\mathrm{u}_{1}^{0}(\underline{\mathrm{x}})\right]=\mathrm{n}_{\mathrm{j}}(\underline{\mathrm{x}}) \mathrm{C}_{\mathrm{ijkl}} \partial_{\mathrm{k}} \mathrm{u}_{1}^{(2)}(\underline{\mathrm{x}}) \\
\text { 5. } & & \operatorname{Lim}_{\|\underline{\mathrm{x}}\| \rightarrow \infty}\left[\mathrm{u}_{\mathrm{i}}^{(2)}(\underline{\mathrm{x}})-\mathrm{E}_{\mathrm{ij}}^{\infty} \mathrm{x}_{\mathrm{j}}\right]=0
\end{array}
$$

En considérant maintenant un tenseur $\underline{\underline{P}}$ symétrique et à valeurs propres strictement positives, on peut procéder à la même transformation que ci-dessus sur la variable $\underline{x}$ et les inconnues $\underline{u}^{(1)}$ et $\underline{u}^{(2)}$. On trouvera alors que les nouvelles inconnues $\underline{\tilde{u}}^{(1)}$ et $\underline{\widetilde{u}}^{(2)}$ en fonction de la variable $\underline{\tilde{x}}$, vérifient les mêmes équations que ci-dessus avec des

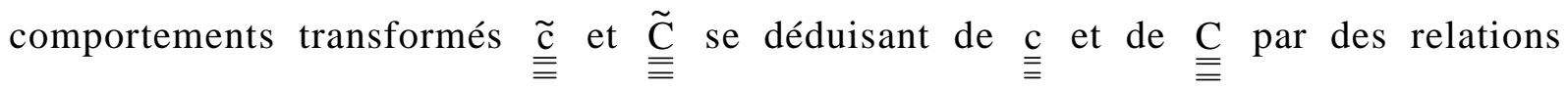
analogues à (12) et avec les données : $\underline{\tilde{u}}^{0}=\underline{\underline{\mathrm{P}}} \cdot \underline{\underline{u}}^{0}$ ou $\underline{\underline{\tilde{\varepsilon}}}^{0}=\underline{\underline{\mathrm{P}}} \cdot \underline{\underline{\varepsilon}}^{0} \cdot \underline{\underline{\mathrm{P}}}$ et $\underline{\underline{\tilde{\mathrm{E}}}}^{\infty}=\underline{\underline{\mathrm{P}}} \cdot \underline{\underline{\mathrm{E}}}^{\infty} \cdot \underline{\underline{\mathrm{P}}}$.

Dans le cas particulier où $\Omega$ est de forme ellipsoïdale, $\underline{\underline{E}}^{\infty}=0$ et $\underline{\varepsilon}^{0}$ est uniforme dans $\Omega$, un résultat classique permet d'affirmer que la déformation dans l'inclusion, notée $\underline{\underline{\varepsilon}}^{(1)}$, est également uniforme et fonction linéaire de $\underline{\underline{\varepsilon}}^{0}$. Si, de plus, $\underline{\underline{\underline{c}}}=\underline{\underline{\underline{\underline{C}}}}$, ce résultat s'écrit sous la forme :

$$
\underline{\underline{\varepsilon}}^{(1)}=\underline{\underline{\underline{S}}}_{\underline{\underline{\varepsilon}}}^{\underline{\underline{\varepsilon}}}
$$


où $\underset{\underline{\underline{S}}}{\underline{\underline{S}}}$ est appelé le «tenseur d'Eshelby ». Dans ce cas, le problème transformé répond également aux mêmes conditions et on peut montrer que le tenseur d'Eshelby $\underline{\underline{\underline{\underline{S}}}}$ de ce problème est relié à $\underset{\equiv}{S}$ par :

$$
\tilde{\mathrm{S}}_{\mathrm{ijkl}}=\mathrm{P}_{\mathrm{im}} \mathrm{P}_{\mathrm{jn}} \mathrm{S}_{\mathrm{mnpq}} \mathrm{Q}_{\mathrm{pk}} \mathrm{Q}_{\mathrm{q} l}
$$

Si l'inclusion est de forme ellipsoïdale, et si la matrice est de comportement isotrope ou transversalement isotrope, on connaît alors pour $\underset{\underline{\underline{S}}}{\underline{S}}$ des expressions analytiques ou semi-analytiques, faisant appel à des intégrales elliptiques ([1],[2],[3]). La relation (20) et le fait que la transformée d'un domaine $\Omega$ ellipsoïdal est également ellipsoïdale permettent d'étendre ces résultats à des cas plus larges de comportement de matrice : il s'agit de comportements pouvant se transformer en un comportement isotrope ou d'isotropie transverse. Ces comportements seront étudiés dans les paragraphes suivants.

La transformation du problème de l'inclusion peut aussi être effectuée dans l'objectif de ramener la géométrie ellipsoïdale à une géométrie sphérique. En effet, si les vecteurs unitaires $\underline{\mathrm{e}}^{\alpha}(\alpha=1,2,3)$ représentent les directions principales de l'ellipsoïde $\Omega$, et les a ${ }^{\alpha} \mathrm{R}$, où $\mathrm{a}^{\alpha}(\alpha=1,2,3)$ sont des nombres strictement positifs et $\mathrm{R}$ une longueur, représentent les demi-diamètres de l'ellipsoïde, on peut définir :

$$
\underline{\mathrm{P}}=\sum_{\alpha} \frac{1}{\mathrm{a} \alpha} \underline{\mathrm{e}}^{\alpha} \otimes \underline{\mathrm{e}}^{\alpha}
$$

Dans le problème transformé, $\tilde{\Omega}$ sera alors une sphère de rayon R. La géométrie sphérique peut être plus intéressante pour des études analytiques. Elle simplifie également les calculs dans le cas de méthodes numériques ne se préoccupant pas d'une forme particulière de $\underline{\underline{\underline{C}}}$, celles, par exemple, fondées sur l'utilisation de la transformation de Fourier [3].

\section{Comportements transformés du cas isotrope}

Etudions maintenant le cas des tenseurs $\underline{\underline{\underline{C}}}$ pouvant se déduire d'un comportement isotrope. Soit $\underset{\underline{\underline{\underline{C}}}}{\underline{\tilde{C}}}$ le tenseur d'élasticité isotrope de coefficients de Lamé $\tilde{\lambda}$ et $\tilde{\mu}$. Le tenseur $\underline{\underline{\underline{\underline{C}}}}$ qui lui correspond par la relation (12) s'écrit sous la forme :

$$
\mathrm{C}_{\mathrm{ijk}}=\tilde{\lambda} \mathrm{H}_{\mathrm{ij}} \mathrm{H}_{\mathrm{kl}}+\tilde{\mu}\left(\mathrm{H}_{\mathrm{ik}} \mathrm{H}_{\mathrm{jl}}+\mathrm{H}_{\mathrm{il}} \mathrm{H}_{\mathrm{jk}}\right)
$$

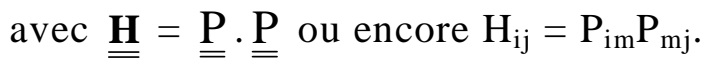

\footnotetext{
* There is a misprint for this equation in the original paper C.R. Acad. Sci. Paris.
} 
Le comportement ainsi défini est orthotrope : une base propre de $\underline{\underline{P}}$ constitue une base d'orthotropie pour $\underset{\underline{\underline{C}}}{\mathrm{C}}$. Plaçons-nous dans une telle base, notons $\mathrm{a}^{\alpha}(\alpha=1,2,3)$ les valeurs propres de $\underline{\underline{\mathrm{P}}}$ dans cette base et posons :

$$
(\alpha=1,2,3, \text { pas de sommation sur } \alpha) \quad \mathrm{c}_{\alpha \alpha}=(\tilde{\lambda}+2 \tilde{\mu})\left(\mathrm{a}_{\alpha}\right)^{4}
$$

$$
\text { et : } \quad \eta=\tilde{\lambda} /(\tilde{\lambda}+2 \tilde{\mu})
$$

En adoptant alors la notation matricielle de Voigt, la matrice des complaisances élastiques correspondant au comportement (22) s'écrit sous la forme :

$$
\mathbf{c}=\left[\begin{array}{cccccc}
\mathrm{c}_{11} & \eta \mathrm{b}_{12} & \eta \mathrm{b}_{13} & & & \\
\eta \mathrm{b}_{12} & \mathrm{c}_{22} & \eta \mathrm{b}_{23} & & & \\
\eta \mathrm{b}_{13} & \eta \mathrm{b}_{23} & \mathrm{c}_{33} & & & \\
& & & \gamma \mathrm{b}_{23} & & \\
& & & & \gamma \mathrm{b}_{31} & \\
& & & & & \gamma \mathrm{b}_{12}
\end{array}\right] \quad \text { où: } \begin{gathered}
\mathrm{b}_{\alpha \beta}=\sqrt{\mathrm{c}_{\alpha \alpha} \mathrm{c}_{\beta \beta}} \\
1 \leq \alpha \leq 3,1 \leq \beta \leq 3 \\
\gamma=(1-\eta) / 2 \\
\end{gathered}
$$

Il s'agit donc d'une famille de comportements orthotropes dépendant de 4 paramètres indépendants que l'on peut choisir comme étant les trois complaisances élastiques $\mathrm{c}_{11}$, $c_{22}, c_{33}$, et le coefficient $\eta$.

L'intérêt de cette famille est de permettre de tenir compte de 3 modules $c_{\alpha \alpha}$ différents dans les trois directions. Elle constitue aussi une bonne approximation pour certains matériaux. Nous avons donné dans le tableau suivant les complaisances élastiques mesurées pour le Niobate de baryum et de sodium qui est un cristal orthotrope (système orthorhombique). L'approximation présentée dans la seconde ligne du tableau a été obtenue en prenant les valeurs exactes de $c_{11}, c_{22}$ et $c_{33}$ et en prenant $\eta=$ $\left[c_{12} c_{23} c_{31} /\left(c_{11} c_{22} c_{33}\right)\right]^{1 / 3}=0,324$. Les valeurs obtenues pour les autres coefficients suivent sensiblement les valeurs mesurées. On a également présenté les coefficients mesurés pour une céramique (système hexagonal) qui a un comportement isotrope transverse (les valeurs avec * sont déduites des autres valeurs du tableau). Les valeurs obtenues par l'approximation ci-dessus $(\eta=0,577)$ ne s'écartent pas de plus $4 \%$ des valeurs mesurées. Grâce à cette approximation, certains calculs relatifs à ce cristal peuvent être menés à l'aide d'un modèle isotrope (dans le problème transformé) ou d'une fonction de Green plus simple que celle de l'isotropie transverse (voir le paragraphe suivant). 


\begin{tabular}{crrrrrrrrr}
\hline $\mathrm{c}_{\alpha \alpha}\left(10^{10} \mathrm{~N} / \mathrm{mm}^{2}\right)$ & $\mathrm{c}_{11}$ & $\mathrm{c}_{22}$ & $\mathrm{c}_{33}$ & \multicolumn{1}{c}{$\mathrm{c}_{44}$} & $\mathrm{c}_{55}$ & $\mathrm{c}_{66}$ & $\mathrm{c}_{12}$ & $\mathrm{c}_{13}$ & $\mathrm{c}_{23}$ \\
\hline $\mathrm{Ba}_{2} \mathrm{NaNb}_{5} \mathrm{O}_{15}$ & 23,9 & 24,7 & 13,5 & 6,5 & 6,6 & 7,6 & 10,4 & 5,0 & 5,2 \\
Approximation & 23,9 & 24,7 & 13,5 & 6,2 & 6,1 & 8,2 & 7,87 & 5,8 & 5,9 \\
Céramique PZT-4 & 13,9 & $13,9^{*}$ & 11,5 & 2,56 & $2,56^{*}$ & $3,05^{*}$ & 7,8 & 7,4 & $7,4^{*}$ \\
Approximation & 13,9 & 13,9 & 11,5 & 2,67 & 2,67 & 2,94 & 8,0 & 7,3 & 7,3 \\
\hline
\end{tabular}

Tableau 1 : Complaisances élastiques de deux cristaux (réf. [4]) et leur approximation par le modèle (25)

Table 1 : Elastic compliances of two crystals (ref. [4]) and their approximation by the model (25).

\section{Application aux fonctions de Green}

Considérons un milieu élastique infini de comportement $\underset{\equiv}{\mathrm{C}}$ soumis à une force ponctuelle $\underline{\phi}$ au point $\underline{x}_{0}$ :

$$
\underline{\mathrm{F}}(\underline{\mathrm{x}})=\underline{\phi} \delta\left(\underline{\mathrm{x}}-\underline{\mathrm{x}}_{0}\right)
$$

La fonction de Green $\underline{\underline{G}}\left(\underline{\mathrm{x}}-\underline{\mathrm{x}}_{0}\right)$ permet d'écrire le champ de déplacement associé à cette force sous la forme :

$$
\underline{\mathrm{u}}(\underline{\mathrm{x}})=\underline{\underline{\mathrm{G}}}\left(\underline{\mathrm{x}}-\underline{\mathrm{x}}_{0}\right) \cdot \underline{\phi}
$$

Le problème transformé par un tenseur $\underline{\underline{P}}$ correspond à un milieu élastique infini de comportement $\underset{\underline{\underline{C}}}{\tilde{C}}$ donné par (12) et soumis au champ de force ponctuelle : avec :

$$
\underline{\tilde{\mathrm{F}}}(\underline{\tilde{x}})=\underline{\mathrm{P}}^{-1} \cdot \underline{\mathrm{F}}(\underline{\mathrm{x}})=\underline{\underline{P}}^{-1} \cdot \underline{\phi} \delta\left(\underline{\mathrm{x}}-\underline{\mathrm{x}}_{0}\right)=\underline{\underline{\mathrm{P}}}^{-1} \cdot \underline{\phi} \operatorname{det}\left(\underline{\underline{P}}^{-1}\right) \delta\left(\underline{\tilde{\mathrm{x}}}-\underline{\tilde{x}}_{0}\right)=\underline{\tilde{\phi}} \delta\left(\underline{\tilde{\mathrm{x}}}-\underline{\tilde{x}}_{0}\right)
$$

$$
\underline{\tilde{\phi}}=\operatorname{det}\left(\underline{\underline{P}}^{-1}\right) \underline{\underline{P}}^{-1} \cdot \underline{\phi}
$$

Le déplacement associé à ce problème vérifie $\underline{\underline{u}}(\underline{\tilde{x}})=\underline{\underline{P}} \cdot \underline{u}(\underline{x})$. A l'aide de cette relation, on peut établir la relation suivante entre les fonctions de Green des deux problèmes :

$$
\underline{\underline{\tilde{G}}}\left(\underline{\tilde{x}}-\underline{\tilde{x}}_{0}\right)=\operatorname{det}(\underline{\underline{P}}) \underline{\underline{P}} \cdot \underline{\underline{G}}\left(\underline{x}-\underline{x}_{0}\right) \cdot \underline{\underline{P}}
$$

Cette relation nous permet de calculer la fonction de Green du milieu infini de comportement $\underset{\underline{\underline{\underline{C}}}}{\tilde{C}}$ à partir de celle correspondant à $\underset{\underline{\underline{C}}}{\mathrm{C}}$, ou inversement.

Prenons par exemple le cas où $\underline{\underline{\underline{C}}}$ est donné par (25) et à partir de $\left(\mathrm{c}_{11}, \mathrm{c}_{22}, \mathrm{c}_{33}, \eta\right)$ calculons :

$$
\begin{gathered}
c=\left(c_{11} c_{22} c_{33}\right)^{1 / 3}, \quad \tilde{\mu}=c(1-\eta) / 2, \quad \tilde{v}=\eta /(1+\eta) \\
(\alpha=1,2,3, \text { pas de sommation sur } \alpha) \quad a_{\alpha}=\left(c_{\alpha \alpha} / c\right)^{1 / 4}
\end{gathered}
$$


Soit $\underline{\underline{P}}$ le tenseur diagonal de valeurs propres $\mathrm{a}_{\alpha}$ dans la base considérée. Posons $\underline{\underline{D}}=\underline{\underline{P}}^{-1} \cdot \underline{P}^{-1}$ et $\rho(\underline{x})=\sqrt{\underline{x} \cdot \underline{D} \cdot \underline{x}}=\sqrt{\frac{x_{1}^{2}}{a_{1}^{2}}+\frac{x_{2}^{2}}{a_{2}^{2}}+\frac{x_{3}^{2}}{a_{3}^{2}}}$. La fonction de Green du milieu infini de comportement $\underline{\underline{\underline{C}}}$ s'obtient alors à partir de celle connue pour le cas isotrope et s'écrit :

$$
\mathrm{G}_{\mathrm{ij}}\left(\underline{x}^{-} \underline{\mathrm{x}}_{0}\right)=\frac{\mathrm{D}_{\mathrm{ij}}}{4 \pi \tilde{\mu} \rho\left(\underline{x}_{-}-\underline{x}_{0}\right)}-\frac{1}{16 \pi \tilde{\mu}(1-\tilde{v})} \partial_{\mathrm{ij}} \rho\left(\underline{x}_{-}-\underline{x}_{0}\right)
$$

Si on suppose $c_{11}=c_{22}$ dans (25), on trouve un cas particulier de comportement isotrope transverse. L'expression donnée par (31) pour ce cas peut être comparée à celle déduite de la solution donnée par Pan and Chou [6] pour le cas général de l'isotropie transverse. On relève alors une erreur dans [6] : le membre droit de la relation (18) dans [6], doit être multiplié par $\left(\mathrm{c}_{11} / \mathrm{c}_{33}\right)^{1 / 4}$. Compte tenu de cette correction, on trouve bien la même expression.

En appliquant la même démarche aux solutions connues de fonction de Green pour le cas d'isotropie transverse ([5],[6],[3]), ainsi qu'aux solutions de potentiels de déplacements [7], on peut obtenir des solutions pour des cas de comportements orthotropes plus riches que l'isotropie transverse, ainsi que pour des cas non orthotropes (si l'axe d'isotropie transverse n'est pas une direction propre de $\underline{\underline{\mathrm{P}}}$ ).

Remerciements : L'auteur tient à remercier vivement le Professeur André Zaoui pour ses suggestions qui ont permis de bien orienter ce travail.

\section{Références bibliographiques}

[1] Eshelby J.D., Determination of the elastic filed of an ellipsoidal inclusion and related problems, Proc. Roy. Soc. Lond. A241 , 376-396 (1957)

[2] Kneer G., Über die Berechnung der Elastizitätsmoduln Vielkristalliner Aggregate mit textur, Phys. Stat. Sol.9, 825-838 (1965)

[3] Mura Toshio, Micromechanics of defects in solids, Martinus Nijhoff Publishers, The Hague, (1982).

[4] Dieulesaint E., Royer D., Ondes élastiques dans les cristaux, Masson et cie Editeurs, Paris 1974, p.123.

[5] Kröner E., Das Fundamentalintegral der anisotropen elastischen Differentialgleichungen, Z. Phys., 136, pp. 402-410 (1953)

[6] Pan Y.-C. and Chou T.-W., Point force solution for an infinite transversely isotropic solid, Transactions of the ASME, Journal of Applied Mechanics, 1976, pp. 608-612.

[7] Green A.E. and Zerna W., Theoretical Elasticity, Oxford University Press, London, 1954. 